\title{
Capilaroscopia periungueal e gravidade da esclerodermia sistêmica"
}

Nail fold capillaroscopy and systemic scleroderma severity"

\author{
Thelma L. Skare ${ }^{1}$ \\ Claudine Casnoch ${ }^{3}$
}

\author{
Letícia Esmanhotto $^{2}$ \\ Marilia Barreto Silva ${ }^{4}$
}

\begin{abstract}
Resumo: FunDAMENTOS - A capilaroscopia periungueal tem sido largamente utilizada para diagnóstico de esclerodermia sistêmica. Mais recentemente descobriu-se que também pode predizer o envolvimento de órgãos internos.

Овјетіvо - Verificar se a capilaroscopia periungueal mostra correlação com a gravidade da esclerodermia sistêmica.

MÉTodos - Foram estudados a capilaroscopia periungueal de 14 pacientes com esclerodermia sistêmica quanto ao número médio de capilares dilatados e às áreas de desvascularização; a medida do envolvimento cutâneo pelo índice de Rodnan modificado; e o grau de gravidade da doença segundo escala proposta por Medsger e cols.

RESULTADOS - Os resultados mostraram boa correlação do índice de desvascularização com o grau de gravidade da doença $(p=0.04)$. Não se encontrou correlação entre o aparecimento de dilatação capilar e o grau de gravidade da doença $(\mathrm{p}=0.572)$. O grau de espessamento cutâneo não mostrou correspondência com o grau de dilatação capilar $(\mathrm{p}=0.76)$, embora mostrasse tendência de associação com desvascularização $(\mathrm{p}=0.07)$.

CONCLUSÃo - Os autores concluem que a presença de desvascularização à capilaroscopia periungueal pode ser usada como elemento indicador de maior gravidade da esclerodermia sistêmica.

Palavras-chave: Escleroderma sistêmico; Esclerodermia limitada; Índice de gravidade de doença
\end{abstract}

\begin{abstract}
BACKGROUND - Nail fold capillaroscopy has been largely used in the diagnosis of systemic sclerosis. It has been recently discovered that this test is also able to predict internal organ damage in systemic sclerosis.

OBJECTIVE - This study was carried out to verify whether nail fold capillaroscopy findings are correlated with disease severity.

METHODS - We studied nail fold capillaroscopy findings regarding dilated and avascular areas from 14 patients with systemic sclerosis; degree of skin involvement by means of a modified Rodnan index; and disease severity with the scale proposed by Medsger et al.

RESULTS - The results showed that the number of avascular areas has a good correlation with disease severity $(p=0.043)$, but not with the number of dilated capillaries $(p=0.57)$. The degree of cutaneous thickening was not related to the degree of capillary dilatation $(p=0.76)$, but tended to be associated with avascular areas $(p=0.07)$.

CONCLUSION - The authors conclude that the number of avascular areas in nail fold capillaroscopy could be used as a sign of disease severity in systemic sclerosis.

Keywords: Scleroderma, limited; Scleroderma, systemic; Severity of illness index
\end{abstract}

\footnotetext{
Recebido em 30.04.2007.

Aprovado pelo Conselho Consultivo e aceito para publicação em 27.02.2008

* Trabalho realizado no Serviço de Reumatologia do Hospital Universitário Evangélico de Curitiba (HUEC) - Curitiba (PR), Brasil.

Conflito de interesse: Nenhum / Conflict of interest: None

Suporte financeiro: Nenhum / Financial funding: None

Preceptora do Serviço de Residência em Reumatologia do Hospital Universitário Evangélico de Curitiba (HUEC) - Curitiba (PR), Brasil. Residente do Serviço de Reumatologia do Hospital Universitário Evangélico de Curitiba (HUEC) - Curitiba (PR), Brasil.

Residente do Serviço de Reumatologia do Hospital Universitário Evangélico de Curitiba (HUEC) - Curitiba (PR), Brasil

Preceptora do Serviço de Residência em Reumatologia do Hospital Universitário Evangélico de Curitiba (HUEC) - Curitiba (PR), Brasil. 


\section{INTRODUÇÃO}

A esclerodermia sistêmica ou esclerose sistêmica (ES) é colagenose relativamente rara, mas que cursa com alta morbidade e mortalidade. ${ }^{1}$ A enfermidade é classicamente associada ao fenômeno de Raynaud, o qual pode preceder a doença por vários anos, sobretudo nos casos em que o paciente desenvolve a forma limitada, ou seja, aquela em que o envolvimento cutâneo está limitado a mãos, pés e face. ${ }^{1}$

$\mathrm{O}$ fato de o paciente poder desenvolver a ES depois de muitos anos da presença do fenômeno de Raynaud levou ao desenvolvimento de um método diagnóstico - a capilaroscopia periungueal, na tentativa de predizer futuros casos dessa colagenose. ${ }^{2}$ A técnica consiste no exame do leito capilar periungueal com auxílio de estereomicroscópio, à procura de vasos dilatados e áreas de desvascularização. ${ }^{2-4}$

Os achados capilares da região periungueal, além de úteis para diagnóstico da ES, quando quantificados parecem correlacionar-se com o envolvimento de alguns órgãos internos. Bredemeier e cols., em 2004, demonstraram associação entre as alterações capilaroscópicas e a presença de lesões pulmonares ativas. ${ }^{5}$ Já outras associações com envolvimento de órgãos internos têm demonstrado resultado variável. ${ }^{6-8}$

Este estudo foi feito com o objetivo de verificar se os achados capilaroscópicos de pacientes com ES correspondem ao grau de gravidade geral da doença medida pela escala proposta por Medsger e cols. e ao grau de envolvimento cutâneo medido pelo índice de Rodnan modificado (Rodnan $\mathrm{m}$ ).

\section{METODOLOGIA}

Após aprovação pelo Comitê de Ética em Pesquisas local e consentimento livre e esclarecido dos pacientes, foram estudados 14 portadores de esclerodermia sistêmica do Ambulatório de Reumatologia do HUEC. Destes, nove tinham a forma limitada da doença; três a forma difusa, e dois a forma mista. O diagnóstico da doença foi feito de acordo com os critérios preliminares do Subcomitê de Critérios Diagnósticos e Terapêutica para Esclerodermia da Associação Americana de Reumatologia. ${ }^{9}$ Os pacientes com envolvimento cutâneo acral (braços, pernas e face) foram considerados portadores de forma limitada, e aqueles com envolvimento acral e de tronco, portadores de forma difusa. ${ }^{9}$ Aqueles que apresentavam forma mista tinham superposição com lúpus eritematoso sistêmico.

Os pacientes foram submetidos à capilaroscopia periungueal quantitativa através de estereomicroscópio, utilizando-se o aumento de 10 vezes. Após imersão da área a ser estudada em óleo secante de esmalte, analisou-se o leito periungueal do segundo ao quinto dígito de ambas as mãos, procedendo-se à contagem de áreas de deleção vascular e de aumento de tamanho dos capilares (capilares ectasiados e megacapilares). Foram considerados capilares ectasiados aqueles com diâmetro aumentado de quatro a nove vezes e megacapilares, aqueles com calibre igual ou superior a 10 vezes o normal. Foi considerada área de deleção focal ou avascular a ausência de dois capilares sucessivos. Para graduação da deleção, seguiu-se esta escala: ${ }^{10}$ deleção $0=$ nenhuma área de deleção; deleção 1 = uma ou duas áreas descontínuas; deleção 2 = mais do que duas áreas contínuas; e deleção $3=$ áreas extensas e confluentes de desvascularização.

Após a contagem de áreas de deleção e número de capilares dilatados em cada dedo, foi obtida a média aritmética para cada um desses itens em cada paciente. Assim, obteve-se o número médio de deleções por paciente e outro correspondente à média de capilares ectasiados e megacapilares por paciente.

Os pacientes foram ainda submetidos à quantificação do envolvimento cutâneo pelo índice de Rodnan $\mathrm{m}$. Esse índice consta da soma da graduação de 17 sítios anatômicos, graduados em $0=$ pele normal; 1 = espessamento leve (a pele está espessada, mas ainda consegue ser pinçada); 2 = espessamento moderado (a pele está espessada e não se consegue pinçá-la, mas ainda não está completamente aderida aos planos profundos, podendo-se ainda fazer leve deslizamento); 3 = espessamento intenso (pele bastante espessada, não passível de ser pinçada, aderida a planos profundos, não podendo ser deslizada). ${ }^{11}$ Os locais analisados foram o dorso de dedos e mãos, os braços e antebraços, a face, a região mediana do tórax e do abdômen, as coxas, as pernas e os pés.

O grau de gravidade da doença foi avaliado pela escala sugerida por Medsger e cols., ${ }^{12}$ na qual são graduados nove itens de envolvimento sistêmico que abrangem os aparelhos e sistemas mais comprometidos na ES (Quadro 1).

Os dados obtidos foram submetidos à análise estatística com auxílio do programa Prism (GraphPad, versão 4.0), sendo utilizados estudos descritivos, teste de Fisher para análise de associação entre amostras com dados descontínuos e teste de coeficiente de correlação de Spearmann para dados contínuos. O nível de significância adotado foi de 5\%.

\section{RESULTADOS}

Todos os 14 pacientes estudados eram do sexo feminino com idade entre 32 e 67 anos (média 48,67 $\pm 10,25$ anos). O tempo de doença variou de 36 a 453 meses (média 141,9 \pm 128 meses). O grau de gravidade da ES dos pacientes estudados variou entre três e nove (média de 5,07 $\pm 1,68$ ).

O número médio de ectasias/megacapilares 
QuADro 1: Graduação da gravidade da doença em esclerodermia sistêmica

\section{SINTOMAS GERAIS}
( ) $0=$ Normal
( ) $1=$ Perda peso $5-9,9 \mathrm{~kg} / \mathrm{Ht} 33-36,9 \%$
( ) $2=$ Perda peso $10-14,9 \mathrm{~kg} / \mathrm{Ht} 29-32,9 \%$
( ) $3=$ Perda peso $15-19,9 \mathrm{~kg} / \mathrm{Ht} 25-28,9 \%$
( ) $4=$ Perda peso $>20 \mathrm{~kg} / \mathrm{Ht}<25 \%$

\section{VASCULAR PERIFÉRICO}

( ) $0=$ Normal

( ) 1 = Fenômeno de Raynaud, requerendo uso de vasodilatadores

( ) 2 = Microulceração de polpas digitais

( ) $3=$ Úlcera de polpas digitais

( ) $4=$ Gangrena digital

\section{PELE}
( ) $0=$ ECT 0
( ) $1=$ ECT $1-14$
( ) $2=$ ECT $15-29$
( ) $3=$ ECT 30-39
( ) $4=$ ECT $>40$

\section{ARTICULAÇÕES / TENDÕES}

( ) $0=$ Distância ponta digital-palma $0-0,9 \mathrm{~cm}$

( ) $1=$ Distância ponta digital-palma $1-1,9 \mathrm{~cm}$

( ) 2 = Distância ponta digital-palma $2-3,9 \mathrm{~cm}$

( ) $3=$ Distância ponta digital-palma $4-4,9 \mathrm{~cm}$

( ) $4=$ Distância ponta digital-palma $>5 \mathrm{~cm}$

\section{MUSCULAR}

( ) $0=$ Sem fraqueza proximal

( ) $1=$ Fraqueza muscular proximal leve

( ) 2 = Fraqueza muscular proximal moderada

( ) 3 = Fraqueza muscular proximal intensa

( ) 4 = Fraqueza muscular proximal intensa; necessário auxílio para deambulação

\section{TRATO DIGESTIVO}

( ) $0=$ Normal

( ) 1 = Hipoperistalse do esôfago distal; Hipoperistalse do intestino delgado

( ) 2 = Aperistalse do esôfago distal; uso antibióticos para supercrescimento bacteriano

( ) 3 = Síndrome de malabsorção; episódios de pseudo-obstrução intestinal

( ) $4=$ Necessidade de nutrição parenteral

\section{PULMÕES}

( ) $0=$ Normal

( ) 1 = Difusão CO 70-80\%; CVF 70-80\%; estertores; fibrose radiológica

( ) 2 = Difusão CO 50-69\%; CVF 50-69\%; hipertensão pulmonar leve

( ) 3 = Difusão CO < 50\%; CVF $<50 \%$; hipertensão pulmonar moderada a grave

( ) $4=$ Necessário uso de oxigenioterapia

\section{CORAÇÃO}

( ) $0=$ Normal

( ) 1 = Defeito condução ao ECG; Fração de ejeção VE 45-49\%

( ) $2=$ Arritmia sintomática; Alargamento VD e VE; Fração de ejeção VE 40-44\%

( ) 3 = Fração de ejeção $\mathrm{VE}<40 \%$

( ) 4 = Insuficiência cardíaca congestiva

\section{RINS}

( ) $0=$ Normal

( ) 1 = Creatinina sérica $1,3-1,6 \mathrm{mg} / \mathrm{dl}$; proteinúria ++

( ) $2=$ Creatinina sérica $1,7-2,9 \mathrm{mg} / \mathrm{dl}$; proteinúria $3+/ 4+$

( ) $3=$ Creatinina sérica $>3 \mathrm{mg} / \mathrm{dl}$

( ) $4=$ Necessidade de diálise

PONTUAÇÃO (soma dos pontos obtidos nos nove itens)

$\mathrm{Ht}=$ hematócrito; ECT $=$ espessamento cutâneo total; $\mathrm{CO}=$ monóxido de carbono; $\mathrm{CVF}=$ capacidade vital forçada; $\mathrm{ECG}=$ eletrocardiograma $\mathrm{VD}=$ ventrículo direito; $\mathrm{VE}=$ ventrículo esquerdo.

variou de zero a três (média de 0,71 \pm 0,99) (Gráfico 1). O grau de deleção média variou de zero a dois (média de $0,85 \pm 0,86)$ (Gráfico 2).

Ao se estudar a correlação entre número médio de ectasias e megacapilares por paciente e grau da gravidade da doença pelo coeficiente de Spearmann, obteve-se $\mathrm{r}=-0.16$, com $\mathrm{p}=0.52$. Quando, nesse mesmo contexto, se estudou o grau médio de deleção, obteve-se $r=0.54$ com $p=0.04$. Esta última correlação encontra-se ilustrada no gráfico 3 .

Ao se estudar a correlação entre o índice de Rodnan m e o número médio de ectasias/megacapila- res, obteve-se $r=0.088$ com $p=0.76$. A correlação do índice de Rodnan m com grau médio de deleção mostrou $r=0.48$ com $\mathrm{p}=0.07$.

\section{DISCUSSÃO}

A esclerodermia sistêmica é doença auto-imune de etiologia desconhecida e com evolução variável, podendo progredir de maneira lenta ou rápida. ${ }^{1}$ É o grau de envolvimento de órgãos internos que determina sua mortalidade, principalmente quando se trata de pulmões, coração e rins. ${ }^{1}$

Seu tratamento é voltado para o tipo de órgão 


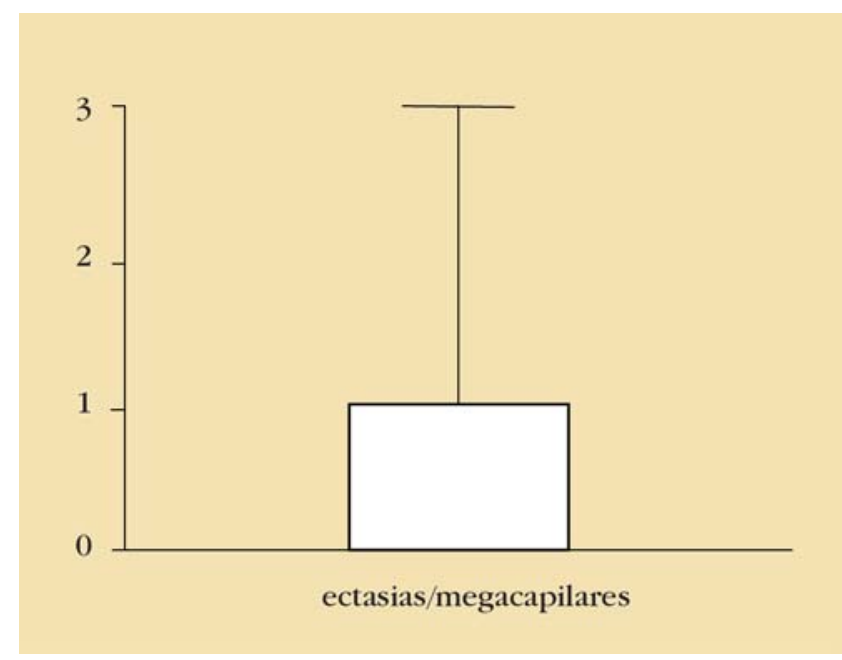

Gráfico 1: Distribuição dos números médios de ectasias/ megacapilares encontrados

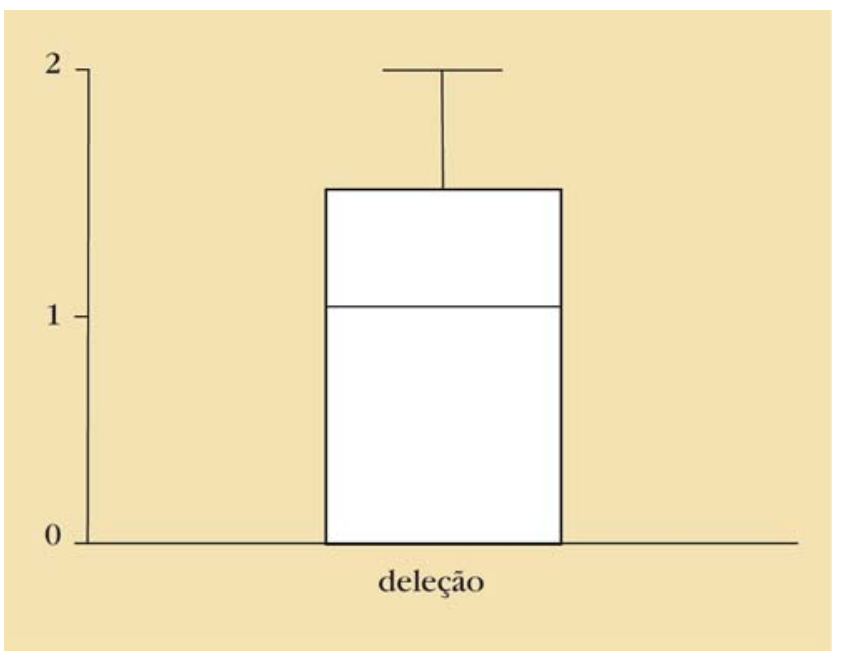

Gráfico 2: Distribuição dos números médios de deleção encontrados

acometido, não existindo uma verdadeira terapia modificadora da enfermidade. ${ }^{13}$ A detecção precoce do envolvimento de determinados sistemas, acompanhada de terapêutica adequada, pode modificar o ritmo de evolução da doença. ${ }^{12}$ Portanto, para o adequado acompanhamento de paciente com ES, é necessária vigilância continua dos órgãos mais freqüentemente envolvidos. Isso pode ser compreendido ao se observar a escala proposta por Medsger e cols., usada para medir a gravidade dessa doença (Quadro 1). Além da coleta de dados clínicos e laboratoriais mais simples, ela demanda a realização de vários exames

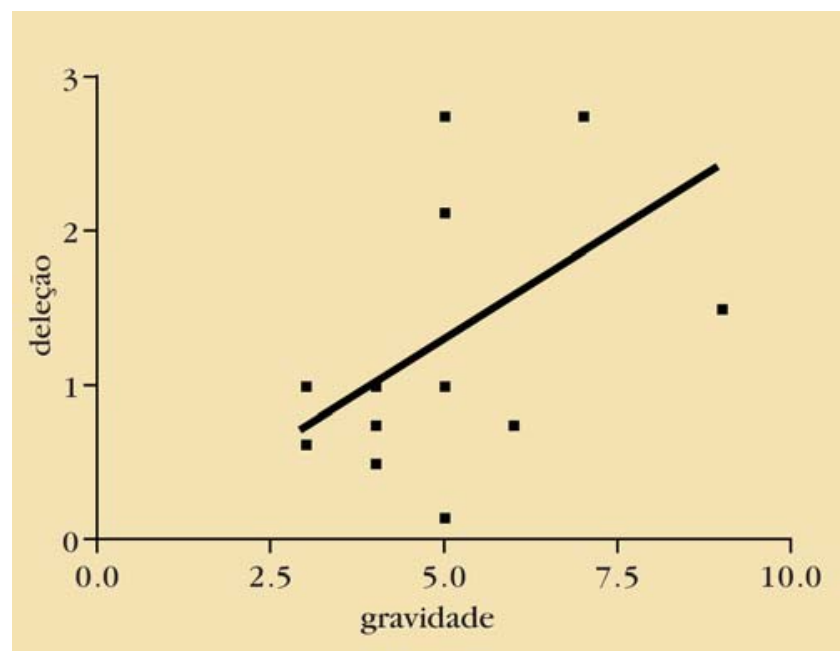

GRÁfico 3: Correlação entre gravidade e número de deleção na capilaroscopia periungueal

complementares mais sofisticados, alguns de alto custo. A repetição periódica desses exames pode ser difícil e dispendiosa senão praticamente impossível em atendimentos na rede pública de saúde de um país como o Brasil.

Neste estudo, a capilaroscopia periungueal, teste barato e acessível, demonstrou que pode ter boa correlação com a gravidade da ES. Isso demonstra seu uso potencial como elemento indicador de gravidade da doença, orientando o médico quais são os pacientes em que é necessário proceder a busca ativa e diligente de complicações. Tal atitude permite um redirecionamento e a otimização dos parcos recursos financeiros da saúde.

No que se refere ao envolvimento cutâneo, embora a desvascularização periungueal não tenha mostrado correlação definitiva, encontrou-se tendência de que seu número médio acompanhe a gravidade de envolvimento cutâneo medido pelo índice de Rodnan $\mathrm{m}$.

Crítica que pode ser feita a este estudo diz respeito ao pequeno número de pacientes analisados, decorrente da relativa raridade dessa doença no país. Por outro lado, ele tem por mérito levantar a questão, para que venha a ser validada por estudos com maior número de casos.

\section{CONCLUSÃO}

Os autores concluem que a capilaroscopia periungueal, ao demonstrar áreas de desvascularização, pode indicar uma forma mais grave da esclerodermia sistêmica, que deve ser acompanhada com maior cuidado pelo médico assistente. 


\section{REFERÊNCIAS}

1. Varga J. Diagnosis and differential diagnosis of systemic sclerosis (scleroderma) in adults. In: Rose B, editor. [Internet]. [updated 2007; cited 2008 Fev 18 ]. Available from: http://www.utdol.com/utd/content/topic.do

2. Cutolo M, Pizzorni C, Sulli A. Capillaroscopy. Best Pract Clin Rheumatol. 2005;19:437-52.

3. Cortes S, Cutolo M. Capillaroscopy patterns in rheumatic diseases. Acta Reumatol Port. 2007;32:29-36.

4. Bukhari M, Hollis S, Moore T, Jayson MI, Herrick AL. Quantitation of microcirculatory abnormalities in patients with primary Raynaud's phenomenon and systemic sclerosis by video capillaroscopy. Rheumatology (Oxford). 2000;39:506-12.

5. Bredemeier M, Xavier RM, Capobianco KG, Restelli VG, Rohde LEP, Pinotti AFF, et al. Capilaroscopia periungueal pode sugerir atividade de doença pulmonar na esclerose sistêmica. Rev Bras Reumatol. 2004; 44:19-30.

6. Simeón CP, Fonollosa V, Vilardell M, Armadans L, Lima $\mathrm{J}$, Cuenca R, et al. Study of the capillary microscopy in scleroderma and their association with organ disease, clinical manifestation and disease progression. Med Clin (Barc). 1991;97:561-4.

7. Puccinelli MLC, Atra E, Sato EI, Andrade LEC. Nailfold capillaroscopy in systemic sclerosis: correlation with involvement of lung and esophagus. Rev Bras Reumatol. 1995;35:136-42.

8. Lovy M, MacCarter D, Steigerwald JC. Relationship between nailfold capillary abnormalities and organ involvement in systemic sclerosis. Arthritis Rheum. 1985;28:496-501.
9. Skare TL. Reumatologia: princípios e prática. 2 ed. Rio de Janeiro: Editora Guanabara Koogan; 2007. p.124-34.

10. Kayser C, Andrade LEC. Capilaroscopia periungueal: importância para a investigação do fenômeno de Raynaud e doenças do espectro da esclerose sistêmica. Rev Bras Reumatol. 2004;44:46-52.

11. Akesson A, Fiori G, Krieg T, van den Hoogen FH, Seibold JR. The assessment of skin, joint, tendon and muscle involvement. Clin Exp Rheumatol. 2003;21 (3 Suppl 29):S5-8.

12. Medsger TA Jr, Silman AJ, Steen VD, Black CM, Akesson A, Bacon PA, et al. A disease severity scale for systemic sclerosis: development and testing. J Rheumatol. 1999; 26:2159-67.

13. Denton CP. Overview of the treatment and prognosis of systemic sclerosis (scleroderma) in adults. In: Rose B, editor . [Internet]. [updated 2007; cited 2008 Fev 18 ]. Available from: http://www.utdol.com/utd/content/topic.do.

ENDEREÇO PARA CORRESPONDÊNCIA / MAILING ADDRESS:

Thelma L. Skare

Rua João Alencar Guimarães, 796

80310-420 - Curitiba - PR

E-mail: tskare@onda.com.br

Como citar este artigo / How to cite this article: Skare TL, Esmanhoto L, Casnoch C, Silva MB. Capilaroscopia periungueal e gravidade da esclerodermia sistêmica. An Bras Dermatol. 2008;83(2):125-9. 